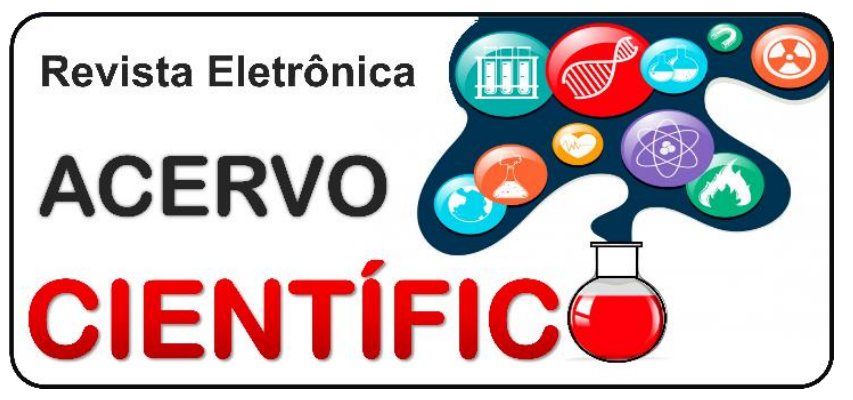

REVISÃO BIBLIOGRÁFICA

Recebido em: 8/2020

Aceito em: 9/2020

Publicado em: 2/2021

\title{
Efeitos da estimulação cerebral profunda em pacientes com doença de Parkinson: uma revisão da literatura
}

\author{
Effects of deep brain stimulation in patients with Parkinson's disease: a literature review
}

Efectos de la estimulación cerebral profunda en pacientes con enfermedad de Parkinson:

una revisión de la literatura

Tainara Sales Miranda ${ }^{1 *}$, Wagner Pablo Corrêa ${ }^{2}$, Alice Fernandes de Almeida Batista ${ }^{3}$, Gabriel de Pádua Walentim Alves ${ }^{3}$, Jorge Steimback Barbosa Junior ${ }^{3}$, Laura Bastos Almeida Vinhal ${ }^{3}$, Letícia Stella Oliveira Pereira ${ }^{4}$, Luiza Fernandes Nonato $^{4}$, Maria Clara Cerqueira Chaves ${ }^{3}$, Taiza dos Santos Azevedo ${ }^{3}$.

Resumo: O presente trabalho trata-se uma revisão de literatura com vistas à avaliação de tal tratamento em seus efeitos sobre sintomas motores e não-motores de pacientes portadores da Doença de Parkinson (DP). Cada paciente deverá ser avaliado individualmente, levando em consideração os sintomas, o tempo de evolução da doença, os efeitos adversos e os possíveis riscos cirúrgicos. Como resultados tem-se que a estimulação cerebral profunda (ECP) regular de alta frequência foi eficiente na correção da bradicinesia. Os efeitos sobre a disfagia não foram claros. A fluência verbal se mostra reduzida no pós-cirúrgico imediato, acreditando-se que esse efeito se deva a microlesões derivadas do implante e não dos sintomas motores. Igualmente, a discinesia e a distonia apresentam melhora após a ECP, ressaltando-se a necessidade de escolha do perfil ideal do paciente para o procedimento: menor de setenta anos, sem problemas psiquiátricos prévios. A pesquisa sobre efeitos dos sintomas não motores, aponta preliminarmente para a segurança do procedimento, embora novos estudos com metodologia e perfil estatístico aprimorados sejam necessários. As perdas cognitivas mínimas no pós-cirúrgico imediato, justificam o procedimento devido à melhor qualidade de vida geral.

Palavras-chave: Estimulação cerebral profunda, Doença de Parkinson, Transtornos motores.

\footnotetext{
Abstract: This paper proposes a literature review in order to evaluate the effects of such treatment in motor, as well as non-motor symptoms of DP patients. Each patient should be evaluated individually, taking into account the symptoms, the time of disease progression, adverse effects and possible surgical risks. It concludes that regular high-frequecy DBS has been efficient in bradykinesia. Effect on dysphagia were nonconclusive. Verbal fluency is reduced immediately after surgery, what has been attributed to implant-caused

${ }^{1}$ Centro Universitário de Caratinga (UNEC), Caratinga - MG. *E-mail: tainarasmiranda@hotmail.com

2 Faculdade de Medicina do Vale do Aço (UNIVAÇO), Ipatinga - MG.

3 Faculdade de Medicina do Mucuri (FAMMUC) da Universidade Federal dos Vales do Jequitinhonha e Mucuri, Teófilo Otoni - MG.

${ }^{4}$ Universidade Vale do Rio Doce (UNIVALE), Ipatinga - MG.
} 
microlesions rather to a motor symptom per se. Similarly, dyskinesia and dystonia have shown improvement after DBS, highlighting a necessity to select the right patient profile for the procedure: individuals under seventy, without prior psychiatric issues. Research on non-motor effects preliminarily points to overall security of the procedure, though new studies with refined methodology and statistical analyses are necessary. Minimum cognitive losses immediately after surgery justify the risk against an overall improvement in patients' quality of life.

Keywords: Deep brain stimulation, Parkinson's disease, Motor disorders.

Resumen: El presente trabajo propone una revisión de literatura para evaluar los efectos del tratamiento sobre los síntomas motores y no-motores de dicha enfermedad. Cada paciente debe ser evaluado individualmente, teniendo en cuenta los síntomas, el tiempo de progresión de la enfermedad, los efectos adversos y los posibles riesgos quirúrgicos. Se concluye por la eficiencia de la DBS regular de alta frecuencia sobre la bradicinesia. Los efectos sobre la disfagia fueron non-conclusivos. La fluidez verbal es reducida en el inmediato pos-quirúrgico, lo que es atribuido a microlesiones causadas por la implantación y no a síntomas motores en si mismos. Discinesia y distonia han mostrado mejora tras DBS, haciéndose hincapié en la necesidad de selección del perfil ideal de paciente: individuos con menos de setenta años y sin problema psiquiátricos previos. La pesquisa sobre efectos no-motores indica una seguridad del procedimiento como un todo, a pesar de que sean necesarios otros estudios con metodologías y análisis estadístico refinados. Las mínimas pérdidas cognitivas en el pos-quirúrgico justifican el procedimiento teniendo en cuenta la mejora de calidad de vida de los pacientes.

Palabras clave: Estimulación cerebral profunda, Enfermedad de Parkinson, Trastornos motores.

\section{INTRODUÇÃO}

A DP descrita pela primeira vez em 1817 por James Parkinson com o nome de Shaking Palsy, é caracterizada pela degradação progressiva de células, prioritariamente dopaminérgicas, presentes na substância negra e pela presença de inclusões intracitoplasmáticas, os corpos de Lewy, causando sintomas motores e não motores, entre os quais destacam-se tremores, rigidez muscular, instabilidade postural e bradicinesia (PARKINSON J, 1817). Atualmente, de acordo com dados da Parkinson's Foundation (2020), mais de 10 milhões de pessoas vivem com essa doença no mundo e estima-se que $4 \%$ dessas pessoas são diagnosticadas antes dos 50 anos.

Segundo dados da World Population Prospects, estima-se que em 2050, um em cada seis habitantes do planeta (16\%) terá mais que 65 anos, contra os atuais um em cada onze habitantes (6\%). Dados da mesma fonte apontam que a projeção é de mais de 400.000 .000 pessoas no mundo tenham idades maiores de oitenta anos. $O$ aumento do número de idosos na população será proporcionalmente acompanhado pelo aumento da incidência de doenças neurodegenerativas entre as quais o Parkinson é uma das principais (ARTEN TLS e HAMDAN AC, 2020). Dessa forma, a pesquisa sobre formas de tratamento em pacientes com DP justifica-se tanto do ponto de vista do planejamento de políticas públicas em saúde, quanto pela melhora das condições de vida dos doentes e de seus familiares.

Diversos medicamentos, inicialmente, foram sugeridos para o tratamento da Doença de Parkinson, como o mercúrio, sugerido pelo próprio James Parkinson e a estricnina, que posteriormente, descobriu-se que traria mais prejuízos que benefícios aos pacientes. Somente com Charcot houve uma real evolução no tratamento da DP, haja vista que os alcalóides antimuscarínicos utilizados por ele, aliviavam os sintomas por algumas horas e foram amplamente indicados até a descoberta dos benefícios do uso de altas doses de Levodopa o final da década de 1960, medicamento utilizado hodiernamente em pacientes com DP (FAHN S, 2014).

A estimulação cerebral profunda ECP é uma inovação no tratamento da DP. Seu uso está relacionado à ativação de regiões cerebrais por meio de eletrodos implantados para tratar distúrbios neurológicos e 
psicológicos, frequentemente reduzindo o uso de medicamentos (BAIG F, et al., 2019). Os eletrodos são implantados em regiões específicas do cérebro que modulam os padrões anormais de movimento e tem sido uma alternativa terapêutica, especialmente para aqueles casos em que os fármacos não controlam, de forma satisfatória, os sintomas motores (MACHADO FA e REPPOLD CT, 2015; HICKEY P e STACY M, 2016).

Neste cenário, o presente artigo tem como objetivo realizar uma revisão bibliográfica a fim de identificar quais são os possíveis efeitos, positivos e negativos, da ECP em pacientes com DP nas funções cognitivas e motoras.

\section{REVISÃO BIBLIOGRÁFICA}

A ECP é um tratamento cirúrgico indicado quando as medidas farmacológicas não produzem alívio suficiente para os pacientes DP, ou quando os efeitos colaterais da terapia medicamentosa medicamentoso se tornam incapacitantes. A ECP pode ser aplicada em diversas regiões do encéfalo, como os Núcleos Subtalâmicos (NST) e Globo Pálido Interno. Entretanto, a estimulação dos NST se torna mais frequente devido à possibilidade de maior redução do tratamento medicamentoso e, consequentemente, dos seus respectivos efeitos colaterais (BARBOSA ENB e CHARCHAT-FICHMAN H, 2019).

\section{Efeitos motores}

\section{Bradicinesia}

A bradicinesia é caracterizada por uma desaceleração dos movimentos associada a rigidez e tremor, sendo considerada um dos sintomas característicos da DP. Tal sintoma está relacionado a um aumento na sincronização das oscilações nas faixas de baixa frequência $(13-30 \mathrm{~Hz})$. Nesse sentido, a terapia de estimulação de alta frequência é uma opção eficaz para pacientes com DP, já que ocorre a modulação da atividade oscilatória anormal nos núcleos subtalâmicos (KÜHN AA, et al., 2008).

Okun MS e Vitek JL (2004) mostram que o benefício terapêutico da ECP alta frequência é muito semelhante à de lesões cirúrgicas realizadas para o tratamento de pacientes parkinsonianos. No entanto, os próprios autores consideram a reversibilidade um ponto positivo da ECP de alta frequência, já que em lesões cirúrgicas, parte do tecido neural é eliminado e no ECP a estimulação pode ser modificada e até mesmo interrompida. Eles ainda evidenciam o fato de que a implantação do eletrodo pode ocorrer de forma bilateral nos núcleos, o que não ocorre em lesões cirúrgicas, já que tal procedimento realizado de forma bilateral está relacionado a frequentes efeitos colaterais indesejáveis.

Dorval AD, et al. (2010) realizaram estudos sobre o tipo de ECP de alta frequência que deve ser utilizada para o tratamento dos sintomas decorrentes da DP, como a bradicinesia. Em um dos seus estudos, foi comparada a ECP regular de alta frequência com a ECP irregular de alta frequência e os resultados evidenciaram que a estimulação irregular de alta frequência, mesmo quando utilizada uma frequência média considerada eficaz, não induziu a melhora da bradicinesia. Já a ECP regular de alta frequência se mostrou eficaz no alívio dos sintomas, já que ocorre uma regularização da atividade dos gânglios basais.

\section{Fluência verbal}

A fluência verbal é o único sinal da DP que apresenta piora significativa após a ECP, como evidenciado por Castelli L, et al. (2006), por meio da comparação de testes de fluência verbal realizados antes e depois do procedimento cirúrgico. As causas dessa piora não foram completamente esclarecidas.

De acordo com Lefaucheur R, et al. (2012), após um prejuízo inicial, há uma recuperação parcial a longo prazo, indicando que, possivelmente, a perda da fluência verbaL não está relacionada diretamente com o estímulo elétrico nos Núcleos Subtalâmicos, mas sim com o procedimento cirúrgico e a evolução natural da DP. Foi demonstrado também que, possivelmente, o comprometimento da fala se deve a microlesões em áreas responsáveis pela função executiva do cérebro e não em áreas motoras.

A hipótese que afirma não haver relação direta entre os estímulos elétricos e a perda da fluência verbal, foi confirmada por Romann AJ, et al. (2018), porém os resultados deste trabalho precisam ser analisados 
com cautela, uma vez que o grupo amostral era pequeno. Além disso, esse estudo indicou que as perdas na fluência verbal podem ser variadas para cada paciente, sendo essencial maiores investigações para que os danos possam ser atenuados por estratégias individualizadas.

\section{Discinesia e distonia}

Além desses, é interessante destacar a discinesia e a distonia, que são movimentos involuntários que podem estar presentes em pacientes com doença de Parkinson. A estimulação cerebral profunda vem sendo utilizada para esses sintomas motores há mais de três décadas e atualmente existem algumas diretrizes que regulamentam esse tratamento, visando a eficácia e a segurança desses métodos.

De acordo com as recomendações da European Federation of Neurological Societies e da Movement Disorder Society, a estimulação cerebral profunda é uma opção de tratamento para melhorar a função motora e a discinesia, e reduzir as flutuações motoras e a utilização de medicamentos. Apesar disso, esse procedimento é recomendado apenas para pacientes que possuem menos do que setenta anos e que não apresentem problemas psiquiátricos e cognitivos avançados, uma vez que há a possibilidade de efeitos adversos, como confusão, hemorragia intracerebral, derrame, convulsões e infecções (FERREIRA JJ, et al., 2013).

Ademais, a European Federation of Neurological Societies aponta a utilização da estimulação cerebral profunda como uma boa opção para o tratamento de distonias primárias, generalizadas e segmentares, e para distonias cervicais, caso abordagens mais conservadoras não funcionem, como o uso de medicamentos e toxina botulínica. Entretanto, deve-se levar em consideração as complicações relacionadas ao procedimento cirúrgico, além de alguns efeitos adversos já relatados, como a bradicinesia em membros que não eram afetados pela distonia. É importante destacar que esse procedimento é menos efetivo em distonias secundárias e que não existe um consenso sobre os tipos de medicamentos e a duração desses tratamentos antes da cirurgia, uma vez que a evidência científica sobre a utilização da estimulação cerebral profunda em casos de distonia é relativamente baixa (ALBANESE A, et al., 2011).

Pesquisas recentes sobre a janela terapêutica, indicam que a realização desse procedimento precocemente pode ser benéfica para alguns pacientes, entretanto, mais estudos devem ser realizados para preencher algumas lacunas do conhecimento, como o tempo ideal para a intervenção cirúrgica, a seleção de qual estrutura será estimulada, os possíveis efeitos adversos e estudos de acompanhamento a longo prazo, para refinar as evidências científicas e aplicar essa técnica de forma segura e eficaz (TODA H, et al., 2016).

\section{Disfagia}

Sabe-se que a disfagia é um sintoma que pode ser presente, independentemente da fase em que se encontra o paciente na DP, e pode prevalecer em $31 \%$ dos que a possuem (CARNEIRO D, et al., 2013). Dessa forma, estudos surgiram acerca dos efeitos da DBS na deglutição. Foi demonstrado que apesar da melhora na compensação orofaríngea durante a alimentação, a deglutição em pacientes, no geral, não apresentou resultados positivos significantes (OLCHIK MR, et al., 2017).

O estudo de Olchik MR, et al. (2017), citado previamente, foi realizado com 10 homens, os quais foram avaliados pré e pós a DBS e possuíam esse sintoma motor. Os resultados mostrados foram que pode haver melhora na disfagia ou não, sendo não-conclusivo, uma vez que 4 destes eram funcionais para essa característica e apresentaram esse sintoma após a DBS, já 3 que possuíam disfagia tornaram-se funcionais. Por fim, para o restante que apresentava disfagia, após esse procedimento, não é notável nenhuma mudança.

Conforme estudos realizados com STN DBS em pacientes com DP, é comprovado que o estágio faríngeo é melhorado, com maior rapidez no tempo de trânsito faríngeo e no grau de liberação do bolo alimentar, mas sem alterações na fase oral da deglutição (CIUCCI MR, et al., 2008). Entretanto, um recente estudo de 20 membros com DP é observado uma redução da frequência de 50 a $25 \%$ da disfagia (KRYGOWSKA-WAJS A, et al., 2016). Dessa forma, a melhora desse sintoma com a DBS ainda apresenta contradições na literatura, mas comprova-se uma melhora no trânsito faríngeo. 


\section{Efeitos não-motores}

Além de sintomas motores, a doença de Parkinson apresenta sintomas não motores associados, que incluem aspectos cognitivos e comportamentais, mudanças em relação ao olfato, distúrbios do sono, entre outros, que aumentam a prevalência da gravidade da doença, portanto, têm impacto negativo na qualidade de vida do paciente (BARBOSA ENB e CHARCHAT-FICHMAN H, 2019).

Nesse sentido, um estudo observacional com dois anos de seguimento, demonstrou benefícios da estimulação profunda sobre os sintomas não motores e a melhora da qualidade de vida dos pacientes após o procedimento, uma vez que está relacionada aos efeitos benéficos significativos sobre a sonolência diurna excessiva, fadiga, problemas de cognição e alucinações e olfato. Ademais, foi observado uma melhora subjetiva da sudorese excessiva e modulação significativa dos sintomas gastrointestinais (DAFSARI HS, et al., 2017). É importante, ainda, citar que a estimulação cerebral profunda é segura em relação à cognição e comportamento, e há estudos que corroboram para melhora da ansiedade, depressão e sobre os transtornos do controle do impulso (KURTIS MM, et al., 2017).

O estudo de Dafsari HS, et al. (2017) usa como metodologia dois questionários, aplicados no pósoperatório e num rastreio 24 meses após a implantação dos eletrodos, um consistindo numa escala de avaliação clínica preenchida pelo médico encarregado do paciente, e a outra numa escala subjetiva preenchida pelos pacientes e baseada em pares antitéticos de opções. Como resultado, os estudos de Dafsari relataram uma percepção clínica de melhora dos sintomas não-motores tanto no pós-operatório quanto no rastreio, enquanto a escala subjetiva relata melhora no pós-operatório e estagnação ou leve declínio no rastreio.

Os autores oferecem a explicação de que a discrepância observada na avaliação subjetiva individual pode se dever ao efeito rebote uma vez cessada a estimulação. São, no entanto, pouco prolíficos em discutir a hipótese de que, uma vez a avaliação clínica não tendo sido feita "às cegas" (unblinded raters), a subjetividade dos clínicos pode ter interferido na medida apresentada por eles gerando um viés de confirmação. Hipóteses alternativas como a de que o contraste entre sintomas não-motores imediatamente antes e imediatamente depois da cirurgia são mais sensíveis e, portanto, podem levar a uma autoavaliação de melhora mais pronunciada nos meses subsequentes e não após 2 anos, não são discutidas e, portanto, o efeito de acomodação subjetiva é ignorado como potencial explicação dos dados apresentados.

\section{Depressão}

Os pacientes com DP podem apresentar sintomas neuropsiquiátricos como ansiedade, impulsividade, introversão, apatia, desesperança, depressão e comportamento suicida em qualquer momento da doença. A depressão é julgada uma das modificações com maior frequência do estado de humor na DP, com uma prevalência de cerca de 40 - 50\%, com depressão maior ocorrendo em 5 a 10\% dos casos (KURTIS MM, et. al., 2017).

\section{Cognição}

A estimulação cerebral profunda em casos de DP pode suscitar efeitos nas funções cognitivas dos pacientes. Alguns dados apontam a estabilidade da cognição após esse procedimento cirúrgico, enquanto outros relatam um declínio leve do raciocínio abstrato, da memória de trabalho e das funções executivas sutis alterações que, geralmente, não são consideradas relevantes clinicamente (KURTIS MM, et al., 2017).

De acordo com Mehanna R, et al. (2017), os resultados conflitantes ocorrem, posto que as pesquisas não utilizam como base casos de DP não tratados cirurgicamente e, constantemente, cuidadores e profissionais da saúde não reportam as alterações cognitivas identificadas nos pacientes. Arten TLS e Hamdam AC (2020) acrescentam que essa divergência pode ser originada, também, pela falta de padronização dos mecanismos utilizados para avaliação da cognição e para comparação dos estudos. Além disso, sabe-se que o agravamento da capacidade cognitiva é um sintoma comum da progressão da DP, uma vez que após 20 anos de acompanhamento, 4 em cada 5 pacientes são identificados com demência (KURTIS MM, et al., 2017). Dessa forma, alguns casos de declínio cognitivo após a estimulação cerebral profunda são atribuídos, essencialmente, ao curso natural da DP (ABBES M, et al., 2018). 
Já de acordo com Cernera S, et al. (2019), cujo trabalho consiste numa revisão descritiva da literatura disponível sobre os efeitos neuropsicológicos da estimulação cerebral profunda, especialmente sobre cognição e funções executivas, as discrepâncias observadas entre trabalhos devem-se à ausência de padronização de metodologia de pesquisa. Nesse sentido, o número e a homogeneidade dos sujeitos de experimento tomados em cada coorte e os diferentes intervalos de rastreio (follow-ups) constituem alguns dos principais elementos de divergência. O padrão identificável nos artigos analisados em tal estudo é o de uma melhora no imediato pós-estimulação, seguido por um declínio ou estabilização em 6 meses. As metodologias empregadas não permitem identificar se as flutuações de medida se devem ao perfil neurocognitivo individual dos pacientes analisados (por exemplo, diferenças educacionais num espaço amostral heterogêneo). Os tempos de rastreio, que, via de regra, quanto mais longos apontam maior declínio de funções executivas, podem se dever à progressão da doença e ao aparecimento de demências relacionadas à doença de Parkinson.

Tal situação é complicada pela não-elucidação da fisiopatologia responsável pelos casos de declínio de funções executivas relatados no pós-operatório, bem como à impossibilidade de afirmar se a melhora cognitiva, nos casos em que acontece, deve-se à estimulação profunda em si ou à diminuição da dose terapêutica de levodopa, que possui efeitos colaterais associados à memória e ao tempo de decisão relatadas na literatura neuropsiquiátrica (CERNERA S, et al., 2019).

Deve-se mencionar, entretanto, que um importante ponto levantado por Cernera S, et al. (2019) refere-se a que, no caso de pacientes que manifestaram piora das funções executivas no imediato pós-operatório, tal fato pode ser explicado por microlesões que afetariam o fluxo de informações núcleo subtalâmico-cortical ou globo pálido interno-cortical. Basiago A e Binder D (2016), concorda com os apontamentos supracitados em relação ao posicionamento dos eletrodos, apontando-o como elemento que pode permitir um controle mais sutil sobre os efeitos obtidos que o tratamento medicamentoso, que afeta receptores no corpo como um todo e não apenas nos tecidos-alvo, argumentando que a sofisticação dos eletrodos, associando-os a controles mentais voluntários e mecanismos capazes de avaliar parâmetros orgânicos, consistem no futuro da técnica.

Assim, mais pesquisas são indicadas no sentido de analisar variações no espaço de implantação dos eletrodos e suas correlações com as observações clínicas para que se possa estabelecer parâmetros preditivos mais seguros quanto aos resultados. De forma semelhante, estudos em que o espaço amostral reúna indivíduos com as características mais homogêneas possíveis e com grupo-controle são indicados, por tratarem de problemas significantes das pesquisas atualmente disponíveis.

Nos efeitos não motores também se observa flutuações motoras. Essas flutuações possuem relação com o uso prolongado de levodopa. Um estudo acompanhou 40 pacientes de Parkinson não motor (NMF) submetidos à STN DBS em 1 ano, e os efeitos foram divididos em quatro grupos: cognitivo, psiquiátrico, autonômico e flutuações sensoriais. O NMF que apresentou mais resultados foi o sensorial e dor com $84,2 \%$ e os domínios cognitivos com diminuição de 60 e $70 \%$ na gravidade. Porém, as flutuações psiquiátricas pareciam responder menos no pós-operatório. O benefício do DBS em NMSB e NMF precisam ser confirmados em estudos controlados (KURTIS MM, et al., 2017).

\section{CONSIDERAÇÕES FINAIS}

A estimulação cerebral profunda vem sendo utilizada nas últimas três décadas e estudos que avaliam a sua eficácia e possíveis efeitos adversos são imprescindíveis para preencher as lacunas do conhecimento. Atualmente, essa técnica é recomendada para o tratamento de alguns sintomas, como os relacionados à função motora, desde que abordagens mais conservadoras não tenham funcionado. Entretanto, outros sintomas, como a fluência verbal, apresentaram piora significativa. Por esse motivo, cada paciente deverá ser avaliado individualmente, levando em consideração os sintomas, o tempo de evolução da doença, os efeitos adversos e os possíveis riscos cirúrgicos, principalmente em pacientes que possuam idade superior a setenta anos. 


\section{REFERÊNCIAS}

1. ABBES M, et al. Subthalamic stimulation and neuropsychiatric symptoms in Parkinson's disease: results from a long-term follow-up cohort study. Journal of Neurology, Neurosurgery \& Psychiatry, 2018; 89:836-843.

2. ALBANESE A, et al. EFNS guidelines on diagnosis and treatment of primary dystonias. European Journal of Neurology, 2011; 18: 5-18.

3. ARTEN TLS, HAMDAN AC. Executive Function in Parkinson's Disease with and without Deep Brain Stimulation. Dementia e Neuropsychologia, 2020; 14(2): 178-185.

4. BAIG F, et al. Deep brain stimulation: practical insights and common queries. Practical Neurology, 2019; 19: 502507.

5. BARBOSA ENB e CHARCHAT-FICHMAN H. Systematic review of neuropsychological instruments used in subthalamic nucleus deep brain stimulation in Parkinson's disease patients. Dement. neuropsychology, 2019; 13(2):162-171.

6. BASIAGO A, BINDER KD. Effects of deep brain stimulation in autonomic function. Brain Sciences, 2016; 6(3).

7. CARNEIRO D, et al. Qualidade de Vida em Disfagia: Uma revisão sistemática. Revista CEFAC, 2013 ; 15:13471356.

8. CASTELLI L, et al. Chronic deep brain stimulation of the subthalamic nucleus for Parkinson's disease: effects on cognition, mood, anxiety and personality traits. European Neurology, 2006;55(3):136-144.

9. CERNERA S, et al. A review of cognitive outcomes across movement disorder patients undergoing deep brain stimulation. Frontiers in Neurology, 2019; 10:419.

10. CIUCCI MR, et al. Subthalamic nucleus deep brain stimulation improves deglutition in Parkinson's disease. Journal of Moviment Disorders, 2008; 23, 676-683.

11. DAFSARI HS, et al. Nonmotor Symptoms Evolution During 24 Months of Bilateral Subthalamic Stimulation in Parkinson's Disease. Movement Disorders, 2017; 33(3): 421-430.

12. DORVAL AD, et al. Deep Brain Stimulation Alleviates Parkinsonian Bradykinesia by Regularizing Pallidal Activity. Journal of Neurophysiology, 2010; 104: 911-921.

13. FAHN S. The medical treatment of Parkinson disease from James Parkinson to George Cotzias. Movement Disorders, 2015;30(1):4-18.

14. FERREIRA JJ, et al. Summary of the recommendations of the EFNS/MdS-ES review on therapeutic management of Parkinson's disease. European Journal of Neurology, 2013; 20: 5-15.

15. HICHEY P, STACY M. Deep Brain Stimulation: A Paradigm Shifting Approach to Treat Parkinson's Disease. Frontiers in Neuroscience, 2016; 10:173.

16. KRYGOWSKA-WAJS A, et al. The effect of subthalamic deep brain stimulation on gastric motility in Parkinson's disease. Parkinsonism \& Related Disorders, 2016; 26: 35-40.

17. KÜHN AA, et al. High-Frequency Stimulation of the Subthalamic Nucleus Suppresses Oscillatory $\beta$ Activity in Patients with Parkinson's Disease in Parallel with Improvement in Motor Performance. Neurobiology of Disease, 2008; 28(24): 6165-6173.

18. KURTIS MM, et al. The effect of deep brain stimulation on the non-motor symptoms of Parkinson's disease: a critical review of the current evidence. NPJ Parkinson's Disease, 2017; 12(3):16024.

19. LEFAUCHEUR R, et al. Early verbal fluency decline after STN implantation: is it a cognitive microlesion effect? Journal of the Neurological Sciences, 2012;321(1-2):96-9.

20. MACHADO FA, REPPOLD CT. The effect of deep brain stimulation on motor and cognitive symptoms of Parkinson's disease: A literature review. Dementia \& neuropsychologia, 2015; 9(1): 24-31.

21. MEHANNA R, et al. Cognitive Impact of Deep Brain Stimulation on Parkinson's Disease Patients. Hindawi Parkinson's Disease, 2017; 15: 3085140.

22. OKUN MS, VITEK JL. Lesion therapy for Parkinson's disease and other movement disorders: Update and controversies. Movement Disorders, 2004; 19(4): 375-89.

23. OLCHIK M, et al. The impact of deep brain stimulation on the quality of life and swallowing in individuals with Parkinson's disease. International Archive of Otorhinolaryngoly, 2018; 22(2): 125-129.

24. PARKINSON'S FOUNDATION, 2020. Disponível em: https://www.parkinson.org/UnderstandingParkinsons/Statistics. Acesso em: 8 jul. 2020.

25. PARKINSON J. An Essay on the Shaking Palsy. Londres: Sherwood, Neely, and Jones, 1817.

26. ROMMAN AJ, et al. Different outcomes of phonemic verbal fluency in Parkinson's disease patients with subthalamic nucleus deep brain stimulation. Arquivos de Neuro-psiquiatria, 2017;75(4):216-220.

27. TODA H, et al. Update on Deep Brain Stimulation for Dyskinesia and Dystonia: A Literature Review. Neurologia Medico Chirurgica, 2016; 56: 236-248. 